\title{
Benzodiazepine-Associated Carcinogenesis: Focus on Lorazepam-Associated Cancer Biomarker Changes in Overweight Individuals
}

\author{
Shih-Chieh Ku1, Pei-Shen Ho', Yu-Ting Tseng'1, Ta-Chuan Yeh², Shu-Li Cheng ${ }^{3}$, and Chih-Sung Liang ${ }^{1,4} \bowtie$ \\ 'Department of Psychiatry, Beitou Branch, Tri-Service General Hospital, School of Medicine, National Defense Medical Center, Taipei, Taiwan, ROC \\ ${ }^{2}$ Department of Psychiatry, Tri-Service General Hospital, School of Medicine, National Defense Medical Center, Taipei, Taiwan, ROC \\ ${ }^{3}$ Department of Nursing, Mackay Medical College, Taipei, Taiwan, ROC \\ ${ }^{4}$ Graduate Institute of Medical Sciences, School of Medicine, National Defense Medical Center, Taipei, Taiwan, ROC
}

\begin{abstract}
Objective Cellular, animal, and human epidemiological studies suggested that benzodiazepines increase the risk of cancer and cancer mortality. Obesity is also clearly linked to carcinogenesis. However, no human studies have examined benzodiazepine-associated carcinogenesis as assessed by changes in cancer biomarkers.

Methods A total of 19 patients were recruited, and received a 6-week treatment of $0.5 \mathrm{mg}$ lorazepam. The measured cancer biomarkers were angiopoietin-2 (ANG-2), soluble CD40 ligand, epidermal growth factor, endoglin, soluble Fas ligand (sFASL), heparin-binding EGF-like growth factor (HB-EGF), insulin-like growth factor binding protein, interleukin (IL)-6, IL-8, IL-18, plasminogen activator inhibitor (PLGF), placental growth factor, transforming growth factor (TGF)- $\alpha$, tumor necrosis factor (TNF)- $\alpha$, urokinase-type plasminogen (uPA), vascular endothelial growth factor (VEGF)-A, VEGF-C, and VEGF-D.

Results Six cancer biomarkers were significantly increased in all patients as a whole. The subgroup analysis revealed a distinct pattern of change. Overweight patients showed a significant increase in 11 cancer biomarkers, including ANG-2, sFASL, HB-EGF, IL-8, PLGF, TGF- $\alpha$, TNF- $\alpha$, uPA, VEGF-A, VEGF-C, and VEGF-D. However, normal-weight patients did not show any changes in cancer biomarkers.

Conclusion Adiposity may have primed the carcinogenic potential, leading to lorazepam-associated carcinogenesis in overweight patients. Epidemiological studies addressing this issue should consider the potential modulator contributing to benzodiazepine-associated carcinogenesis.

Psychiatry Investig 2018;15(9):900-906
\end{abstract}

Key Words Benzodiazepines, Cancer, Carcinogenesis, Lorazepam, Overweight.

\section{INTRODUCTION}

Benzodiazepines refer to a chemical structure consisting of a benzene ring fused to a diazepine ring. ${ }^{1}$ Binding on the gamma amino butyric acid A receptor, benzodiazepines work as a positive allosteric modulator. ${ }^{1}$ Although benzodiazepines are considered a class of psychoactive drugs, they are commonly used as sedative-hypnotic, anesthetic, anti-

Received: January 25, 2018 Revised: April 16, 2018

Accepted: May 2, 2018

$\bowtie$ Correspondence: Chih-Sung Liang, MD

Department of Psychiatry, Beitou Branch, Tri-Service General Hospital, National Defense Medical Center, No. 60, Xinmin Road, Beitou District, Taipei 112, Taiwan, ROC

Tel: +886-2-2895-9808, Fax: +886-2-2895-7633, E-mail: lcsyfw@gmail.com

(c) This is an Open Access article distributed under the terms of the Creative Commons Attribution Non-Commercial License (http://creativecommons.org/licenses/bync/4.0) which permits unrestricted non-commercial use, distribution, and reproduction in any medium, provided the original work is properly cited. convulsant, and muscle relaxant drugs. Because of the wide therapeutic index, benzodiazepines have largely replaced barbiturates and continue to be one of the most consumed and highly prescribed classes of drugs for the treatment of insomnia and anxiety disorders.

The adverse effects of benzodiazepine represent a substantial public health concern. These include daytime fatigue, decreased cognitive function, tolerance, dependence, impaired immune system, and increased risk of mortality. Benzodiazepine-associated excess mortality has been linked to the potential for carcinogenesis. ${ }^{2}$ Preliminary evidence from animal studies suggested that benzodiazepines increased the risk of thyroid cancer ${ }^{3}$ and liver cancer. ${ }^{4}$ A large cohort study using longitudinal electronic medical records found a significant elevation of cancer incidence among benzodiazepine users, even among users with low cumulative exposure. ${ }^{5}$ Adding credibility to the carcinogenesis hypothesis of benzodiaze- 
pine, a recent study using the National Health Insurance Research Database suggested that several benzodiazepines have a high risk for cancer development, including clonazepam, lorazepam, alprazolam, bromazepam, zolpidem, and zopiclone (zolpidem and zopiclone are known as the "Z-drugs," or benzodiazepine receptor agonists). ${ }^{6}$ However, the question of the carcinogenic potential of benzodiazepines is still being debated. In vitro laboratory studies indicated that benzodiazepines might inhibit the proliferation of pituitary tumor cells ${ }^{7}$ and showed antitumor effects on breast adenocarcinoma cells. ${ }^{8}$ Another study using a nationwide approach failed to show a link between benzodiazepine use and cancer risk. ${ }^{9}$

Importantly, carcinogenesis is a progressive chronic process that includes angiogenesis, cell proliferation, cell metastasis, cell adhesion, apoptosis, and inflammation. ${ }^{10}$ Measuring the biomarkers of these pathophysiological changes after benzodiazepine exposure provides a window into the issue of carcinogenesis of benzodiazepines. To date, no human study has been performed to investigate the biomarkers of carcinogenesis after benzodiazepine exposure. The aim of this study is to examine the cancer biomarkers for benzodiazepine-naïve individuals after taking benzodiazepines. As being overweight has been associated with an increased risk of cancers ${ }^{11}$ and cancer mortality, ${ }^{12}$ this study also addressed whether overweight status contributes to carcinogenic potential in individuals treated with benzodiazepines.

\section{METHODS}

Between January 2017 and June 2017, subjects who were hospitalized for treatment of depression and insomnia at the Beitou Branch of Tri-Service General Hospital, National Medical Defense Center, Taiwan, were considered eligible for participation in the study. Written informed consent was obtained in accordance with the National Health and Medical Research Council guidelines. All participants were fully informed about the aims and details of the study and were free to withdraw their consent at any time. The Institutional Review Board for the Protection of Human Subjects at the TriService General Hospital approved the protocol (IRB No. 1-104-05-101).

To avoid potential confounders, patients were required to be male, drug-naïve, between the ages of 20 and 40, and in good health. The exclusion criteria were as follows: 1) having suffered from or suffering from cancer or a tumor; 2) history of a major medical disorder (e.g., hypertension, diabetes, or rheumatoid arthritis); 3) history of a neurological disorder (e.g., meningitis, epilepsy, or multiple sclerosis); 4) history of a major psychiatric disorder (e.g., bipolar disorder, schizophrenia, or mental retardation); 5) diagnosis of a substance- related disorder; 6) previous head trauma with loss of consciousness; and 7) previous exposure to psychotropic agents (e.g., hypnotics, antidepressants, mood stabilizers, antipsychotics). Tobacco use and alcohol consumption were not allowed during the study period. Patients with major depression were excluded because the likelihood of polypharmacy during the study period was high. All were diagnosed with an adjustment disorder with depressed mood. The diagnosis was made by two certified psychiatrists according to the Diagnostic and Statistical Manual of Mental Disorders, Fifth Edition.

All patients received $0.5 \mathrm{mg}$ of lorazepam at night for the treatment of insomnia for six weeks. If the patient's depressive symptoms required pharmacotherapy, $20 \mathrm{mg}$ of fluoxetine or $10 \mathrm{mg}$ of escitalopram was given. Because epidemiological studies have shown that obesity is associated with increased risk of several cancer types, ${ }^{11,13}$ the patients' body mass index (BMI) was recorded at the baseline (W0) and at the end of study (W6).

The procedures for collection, preparation, freezing, and thawing of all serum samples used in this study were performed in a highly consistent manner, including the collection and handling of commercial samples. After fasting, peripheral venous blood samples were collected in the morning between 8:00 and 9:00 by venipuncture from patients prior to and after a 6-week treatment with lorazepam. For the preparation of the serum samples, $10 \mathrm{~mL}$ of peripheral blood was drawn into a Vacutainer Gel and Clot Activator tube and centrifuged to separate the serum, which was then aliquoted into NUNC-cryovial tubes and stored at $-84^{\circ} \mathrm{C}$. None of the serum samples had been previously thawed prior to the thawing for the Luminex assay.

The serum levels of human cancer markers were measured by using Luminex, which is a suspension assay that combines the principles of a standard sandwich immunoassay with flow cytometry, thus allowing a multiplex analysis of up to 100 individual biomolecules in a single microtiter plate well. ${ }^{14}$ The human cancer biomarker panel 18-plex (\#171-AC500M) was used to analyze serum samples (pg/mL) according to the manufacturer's instructions on Luminex analyzers (Bio-Plex 200, Genmall Biotechnology Co., LTD., Taipei, Taiwan). The biomarkers were involved in the process of carcinogenesis, such as angiogenesis, metastasis, cell proliferation, cell adhesion/migration, apoptosis, and inflammation. The measured cancer biomarkers were angiopoietin-2 (ANG-2), soluble CD40 ligand (sCD40L), epidermal growth factor (EGF), endoglin, soluble Fas ligand (sFASL), heparin-binding EGF-like growth factor (HB-EGF), insulin-like growth factor binding protein (IGFBP-1), interleukin (IL)-6, IL-8, IL-18, plasminogen activator inhibitor (PAI-1), placental growth factor 
(PLGF), transforming growth factor (TGF)- $\alpha$, tumor necrosis factor (TNF)- $\alpha$, urokinase-type plasminogen (uPA), and vascular endothelial growth factor (VEGF)-A, VEGF-C, VEGF-D. Standard curves were created from duplicate values, and all samples were analyzed as single determinations. All analyses were performed in one batch using kits from the same production lot. The intra-assay and inter-assay coefficients of variation were below $10 \%$.

The participants were divided into normal-weight and overweight patients, whose BMI exceeded $25 \mathrm{~kg} / \mathrm{m}^{2}$. Group differences in continuous variables were analyzed using independent sample t-tests. Group differences in baseline cancer markers were analyzed with a multivariate general linear model with covariate adjustment. The covariates were age, height, weight, educational levels, and the use of fluoxetine.

The paired sample t-tests were performed to examine within-group differences in cancer markers after lorazepam treatment. For the cancer markers that significantly changed after lorazepam treatment, another multivariate general linear model was created. In this model, changes in the levels of cancer markers were calculated as dependent variables, and antidepressant treatment (fluoxetine or escitalopram) was entered as a nominal variable. Changes in body weight also covaried. Identification of whether the patterns of cancer biomarker changes were different for the two groups was done by examining a multivariate general linear model with a covariate of weight changes. The analyses were considered statistically significant if the $\mathrm{p}$ values were less than or equal to 0.05 (two-tailed). All statistical analyses were conducted using the Statistical Package for the Social Sciences software for Windows ver. 22 (IBM Corp., Armonk, NY, USA).

\section{RESULTS}

Between January 2017 and June 2017, a total of 19 male inpatients were recruited, with a mean age of $26.1 \pm 3.4$ years. The participants were divided into an overweight group $(n=9)$ and a normal-weight group $(n=10)$ according to the BMI cut-off point of $25 \mathrm{~kg} / \mathrm{m}^{2}$. Table 1 shows the clinical and demographic characteristics among all the participants and the two different groups. As expected, the overweight group had significantly higher weight and BMI than those in the normal-weight group. Otherwise, the two groups did not differ from each other at baseline.

Table 2 reveals the baseline cancer biomarkers among all normal patients and the two different groups. The overweight patients did not differ from the normal-weight patients in any of the 18 cancer biomarkers.

Table 3 shows the changes in cancer biomarkers in all patients after a 6-week treatment of lorazepam. The levels of sFASL $(\mathrm{p}=0.034)$, HB-EGF $(\mathrm{p}=0.05), \mathrm{IL}-8(\mathrm{p}=0.013)$, TGF- $\alpha$ $(\mathrm{p}=0.045)$, uPA $(\mathrm{p}=0.001)$, and VEGF-A $(\mathrm{p}=0.028)$ significantly increased after a 6-week treatment of lorazepam. The changes in the other 12 cancer biomarkers did not reach significance. The multivariate general linear model determined that the use of fluoxetine did not predict the changes in the six cancer biomarkers: sFASL $(\mathrm{p}=0.174)$, HB-EGF $(\mathrm{p}=0.418)$, IL- $8(p=0.285)$, TGF- $\alpha(p=0.265), u P A(p=0.058)$, and VEGF-A ( $\mathrm{p}=0.864)$.

Table 4 reveals within-group changes in cancer biomarkers in overweight and normal-weight patients after the 6-week lorazepam treatment. Interestingly, compared with all patients as a whole, the overweight patient revealed more cancer biomarkers that were significantly increased after lorazepam treatment. A total of 11 cancer biomarkers in the overweight patients reached statistical significance, including ANG-2 $(\mathrm{p}=0.003)$, sFASL $(\mathrm{p}=0.005)$, HB-EGF $(\mathrm{p}=0.009)$, IL-8 $(p=0.006)$, PLGF $(p=0.008)$, TGF- $\alpha(p=0.005)$, TNF- $\alpha$ $(p=0.035), u P A(p=0.028)$, VEGF-A $(p=0.049)$, VEGF-C $(\mathrm{p}=0.023)$, and VEGF-D ( $\mathrm{p}=0.011)$. The multivariate general linear model determined that the use of fluoxetine only predicted the changes in HB-EGF $(p=0.04)$ and VEGF-D $(\mathrm{p}=0.016)$ but not in ANG-2 $(\mathrm{p}=0.596)$, sFASL $(\mathrm{p}=0.408)$, IL-8 $(p=0.613)$, PLGF $(p=0.245)$, TGF- $\alpha(p=0.093)$, TNF- $\alpha$ $(\mathrm{p}=0.12)$, uPA $(\mathrm{p}=0.108)$, VEGF-A $(\mathrm{p}=0.549)$, and VEGF-C $(\mathrm{p}=0.224)$. Surprisingly, in the normal-weight patients, none of the cancer biomarkers reached statistical significance.

Figure 1 illustrates the patterns of change in the cancer biomarker after the 6-week lorazepam treatment. A multivariate general linear model confirmed that the overweight

Table 1. Clinical characteristics in overweight and normal-weight patients

\begin{tabular}{|c|c|c|c|c|c|}
\hline & Total $(\mathrm{N}=19)$ & Overweight $(\mathrm{N}=9)$ & Normal-weight $(\mathrm{N}=10)$ & $\mathrm{t}$ & $\mathrm{p}^{*}$ \\
\hline Age (y) & $26.1 \pm 3.4$ & $26.9 \pm 3.3$ & $25.3 \pm 3.5$ & 1.021 & 0.322 \\
\hline Education (y) & $15.6 \pm 1.6$ & $15.4 \pm 1.5$ & $15.7 \pm 1.8$ & 0.337 & 0.74 \\
\hline Height (cm) & $170.7 \pm 5.7$ & $170.9 \pm 6.1$ & $170.5 \pm 5.8$ & 0.155 & 0.879 \\
\hline Weight (kg) & $70.7 \pm 12.8$ & $81 \pm 9$ & $61.5 \pm 7.7$ & 5.09 & $<0.001$ \\
\hline BMI $\left(\mathrm{kg} / \mathrm{m}^{2}\right)$ & $24.2 \pm 3.8$ & $27.7 \pm 2$ & $21.1 \pm 1.7$ & 7.729 & $<0.001$ \\
\hline
\end{tabular}

*differences in characteristics between groups were analyzed with independent-samples t-tests. BMI: body mass index 
Table 2. Baseline cancer markers in overweight and normal-weight patients

\begin{tabular}{|c|c|c|c|c|c|}
\hline & Total $(\mathrm{N}=19)$ & Overweight $(\mathrm{N}=9)$ & Normal-weight $(\mathrm{N}=10)$ & $\mathrm{F}$ & $\mathrm{p}^{*}$ \\
\hline ANG-2 & $583 \pm 177.8$ & $485 \pm 149.2$ & $671.2 \pm 158.7$ & 0.019 & 0.894 \\
\hline sCD40L & $84.1 \pm 15.5$ & $86.6 \pm 18.9$ & $81.9 \pm 12.3$ & 1.807 & 0.204 \\
\hline EGF & $24.9 \pm 5.5$ & $25.9 \pm 6.6$ & $23.9 \pm 4.4$ & 0.855 & 0.373 \\
\hline Endoglin & $647.9 \pm 334.1$ & $761.8 \pm 451.1$ & $545.4 \pm 133.1$ & 0.045 & 0.835 \\
\hline sFASL & $122.9 \pm 20.4$ & $120.4 \pm 24.7$ & $125.1 \pm 16.8$ & 0.348 & 0.566 \\
\hline HB-EGF & $97.4 \pm 15.5$ & $97 \pm 19.8$ & $97.8 \pm 11.7$ & 0.566 & 0.466 \\
\hline IGFBP & $1237 \pm 305$ & $1227 \pm 354$ & $1247 \pm 274$ & 0.505 & 0.491 \\
\hline IL-6 & $23.4 \pm 4$ & $23.6 \pm 4.5$ & $23.1 \pm 3.7$ & 0.056 & 0.817 \\
\hline IL-8 & $5.9 \pm 1.1$ & $5.9 \pm 1.1$ & $6 \pm 1$ & 0.057 & 0.815 \\
\hline IL-18 & $57.2 \pm 25.3$ & $66.4 \pm 30.9$ & $49 \pm 16.6$ & 0.016 & 0.903 \\
\hline PAI & $4046 \pm 1839$ & $4749 \pm 2437$ & $3413 \pm 689$ & 0.078 & 0.785 \\
\hline PLGF & $26.2 \pm 7$ & $26.4 \pm 7.4$ & $26 \pm 7$ & 0.337 & 0.572 \\
\hline TGF- $\alpha$ & $30 \pm 5.9$ & $29.8 \pm 7.1$ & $30.2 \pm 5$ & 0.245 & 0.629 \\
\hline TNF- $\alpha$ & $15.5 \pm 3.3$ & $15.3 \pm 3.6$ & $15.8 \pm 3.2$ & 0.002 & 0.967 \\
\hline $\mathrm{uPA}$ & $461.5 \pm 202.8$ & $425.7 \pm 178.3$ & $493.7 \pm 227$ & 0.065 & 0.803 \\
\hline VEGF-A & $143.5 \pm 28.6$ & $144.9 \pm 32.4$ & $142.4 \pm 26.5$ & 0.025 & 0.877 \\
\hline VEGF-C & $448.9 \pm 85.1$ & $446.5 \pm 91.9$ & $450.9 \pm 83.4$ & 0.085 & 0.776 \\
\hline VEGF-D & $273.9 \pm 53.9$ & $278.3 \pm 74.4$ & $269.9 \pm 29.3$ & 0.114 & 0.741 \\
\hline
\end{tabular}

*group differences in cancer markers were analyzed with a multivariate general linear model with covariates of age, height, weight, educational levels, and the use of fluoxetine. ANG-2: angiopoietin-2, sCD40L: soluble CD40 ligand, EGF: epidermal growth factor, sFASL: soluble Fas ligand, HB-EGF: heparin-binding EGF-like growth factor, IGFBP: insulin-like growth factor-binding protein, IL: interleukin, PAI: plasminogen activator inhibitor, PLGF: placental growth factor, TGF: transforming growth factor, TNF: tumor necrosis factor, uPA: urokinasetype plasminogen activator, VEGF: vascular endothelial growth factor

patients differed from the normal-weight patients in nine cancer biomarkers. The increases in the nine cancer biomarkers were significantly greater, including sFASL $(\mathrm{p}=0.035)$, HB-EGF $(\mathrm{p}=0.045)$, ANG-2 $(\mathrm{p}<0.001)$, VEGF-C $(\mathrm{p}=0.025)$, VEGF-D $(\mathrm{p}=0.013)$, IL-8 $(\mathrm{p}=0.049)$, PLGF $(\mathrm{p}=0.003)$, TGF- $\alpha(\mathrm{p}=0.03)$, and TNF- $\alpha(\mathrm{p}=0.032)$.

\section{DISCUSSION}

This is the first human study to examine the carcinogenic potential of lorazepam through a simultaneous analysis of 18 human cancer biomarkers. Our data showed that the levels of sFASL, HB-EGF, IL-8, TGF- $\alpha$, uPA, and VEGF-A were significantly increased after the 6-week treatment of lorazepam. However, the lorazepam-induced cancer biomarker changes were significantly different for the overweight and the normal-weight patients. In the normal-weight patients, lorazepam did not induce any changes in the cancer biomarkers; however, in the overweight patients, a total of 11 cancer biomarkers were significantly increased, including ANG-2, sFASL, HB-EGF, IL-8, PLGF, TGF- $\alpha$, TNF- $\alpha$, uPA, VEGF-A, VEGF-C, and VEGF-D. The patterns of change in the cancer biomarkers revealed that the slopes were signifi- cantly steeper in the overweight patients. Taking these findings together, lorazepam may not induce carcinogenic potential in normal-weight individuals, while lorazepamassociated carcinogenic potential may be determined in overweight patients.

We suggest that lorazepam may work in concert with adiposity, thereby promoting carcinogenesis. The rise in the numbers of overweight and obese individuals has been substantial and widespread over the past few decades. A large volume of epidemiological evidence points to an association between being overweight and increased risk of several cancer types. ${ }^{11}$ The biological mechanisms underpinning these associations are still not fully understood. Currently, the biological mechanisms that have received the most research attention are sex hormone metabolism, insulin and insulin-like growth factor (IGFs) signaling, adipokine pathophysiology, and subclinical inflammation. ${ }^{15,16}$ The adiposity-cancer link supports our findings that only overweight patients showed significant increases in the cancer biomarkers after six weeks of treatment with lorazepam.

Preclinical and animal research, randomized controlled trials, and epidemiological studies have all reported that benzodiazepines may increase the risk of carcinogenesis and 
Table 3. Changes in cancer markers in all patients after a 6-week lorazepam treatment $(\mathrm{N}=19)$

\begin{tabular}{|c|c|c|c|c|}
\hline & Baseline & Week 6 & $\mathrm{t}$ & $\mathrm{p}^{*}$ \\
\hline ANG-2 & $583 \pm 177.8$ & $595.4 \pm 158.6$ & 0.561 & 0.582 \\
\hline sCD40L & $84.1 \pm 15.5$ & $90.9 \pm 16.5$ & 1.872 & 0.077 \\
\hline EGF & $24.9 \pm 5.5$ & $27.7 \pm 7.9$ & 1.741 & 0.099 \\
\hline Endoglin & $647.9 \pm 334.1$ & $599.2 \pm 251.8$ & 0.563 & 0.58 \\
\hline sFASL & $122.9 \pm 20.4$ & $131.2 \pm 23.7$ & 2.301 & 0.034 \\
\hline HB-EGF & $97.4 \pm 15.5$ & $104.1 \pm 17.7$ & 2.103 & 0.05 \\
\hline IGFBP & $1237 \pm 305$ & $1379 \pm 429$ & 1.869 & 0.078 \\
\hline IL-6 & $23.4 \pm 4$ & $24.5 \pm 4.2$ & 1.439 & 0.167 \\
\hline IL-8 & $5.9 \pm 1.1$ & $6.5 \pm 1.3$ & 2.766 & 0.013 \\
\hline IL-18 & $57.2 \pm 25.3$ & $57.6 \pm 28.2$ & 0.073 & 0.942 \\
\hline PAI & $4046 \pm 1839$ & $3718 \pm 1124$ & 0.634 & 0.534 \\
\hline PLGF & $26.2 \pm 7$ & $28.2 \pm 7.4$ & 1.922 & 0.071 \\
\hline TGF- $\alpha$ & $30 \pm 5.9$ & $32.6 \pm 6.6$ & 2.154 & 0.045 \\
\hline TNF- $\alpha$ & $15.5 \pm 3.3$ & $16.5 \pm 3$ & 1.625 & 0.122 \\
\hline $\mathrm{uPA}$ & $461.5 \pm 202.8$ & $560.3 \pm 256.9$ & 3.83 & 0.001 \\
\hline VEGF-A & $143.5 \pm 28.6$ & $153.7 \pm 26.1$ & 2.394 & 0.028 \\
\hline VEGF-C & $448.9 \pm 85.1$ & $476.8 \pm 73.3$ & 1.85 & 0.081 \\
\hline VEGF-D & $273.9 \pm 53.9$ & $287.8 \pm 60.9$ & 2.032 & 0.057 \\
\hline
\end{tabular}

*differences in cancer markers after six weeks of lorazepam treatment were analyzed with paired-samples t-tests. ANG-2: angiopoietin-2, sCD40L: soluble CD40 ligand, EGF: epidermal growth factor, sFASL: soluble Fas ligand, HB-EGF: heparin-binding EGF-like growth factor, IGFBP: insulin-like growth factor-binding protein, IL: interleukin, PAI: plasminogen activator inhibitor, PLGF: placental growth factor, TGF: transforming growth factor, TNF: tumor necrosis factor, uPA: urokinase-type plasminogen activator, VEGF: vascular endothelial growth factor

cancer mortality. ${ }^{2}$ Attention has been paid to whether differences exist in carcinogenic potential across different benzodiazepines. ${ }^{17}$ Focusing on the carcinogenesis of lorazepam, the findings are inconsistent. Previous basic studies used S. typhimurium, animal cells in vitro, rat bone-marrow cells, and rat liver in vivo and examined the deoxyribonucleic acid strand break, chromosomal aberrations, liver tumor initiation and promotion activity, and a long-term carcinogenesis assay in rats, ${ }^{17}$ all showing negative or equivocal results. However, a recent study reported that lorazepam in oral form exhibited statistically significant genotoxicity in normal human lymphocyte cultures. ${ }^{18}$ A national database study also noted the increased risk of cancer in individuals taking lorazepam. ${ }^{6}$ In the current human in vivo study, lorazepam-associated carcinogenic potential was only observed in overweight patients but not in normal-weight patients. The inconsistent findings between laboratory studies and epidemiologic data may imply that the carcinogenicity of lorazepam in humans could be affected by other modulators such as obesity.

Lorazepam-associated carcinogenesis may be related to the progression of tiny pre-existing cancers rather than from the effects of microscopic cancer initiation. One study analyzed all randomized, placebo-controlled trials for the four drugs-Z-drugs (zaleplon, eszopiclone, and zolpidem)-and ramelteon and reported the incidence of 12 cancers or tumors of uncertain malignancy among 6190 participants receiving the hypnotics and no cases among 2535 participants receiving placebo. ${ }^{19}$ As the duration of these trials were less than six months, the authors suggested that the hypnotic-associated direct carcinogenic potential may play a minor role in these cancer cases. However, in our study, the baseline levels of cancer markers did not differ between the overweight and the normal-weight patients. After 6-week treatment of lorazepam, the overweight patients showed significant increases in 11 cancer biomarkers. This suggests that adiposity may have primed the susceptibility of tumor initiation or promotion of existing microscopic tumor.

The findings of our study should be interpreted against the backdrop of the following limitations. First, our sample size was small, which might limit the widespread application of the findings. Furthermore, the probability of type 1 error may be increased. Using a more stringent alpha level of 0.01 , we still found six cancer biomarkers that were significantly increased after lorazepam treatment in the overweight patients. Second, the participants also received antidepressant treatment, which may confound our findings. However, the multivariate general linear model confirmed that the use of antidepressants did not predict the changes in cancer bio- 
markers. Third, the study duration was short; therefore, whether the changes in cancer biomarkers are transient or gradually increased remains to be investigated. Fourth, changes in biomarkers need to be interpreted in clinical con- text, as cancer biomarkers can also change in other conditions. In addition, increasing biomarkers is not necessarily evidence of carcinogenesis. However, cancer biomarkers are quantifiable traits that may be helpful in early detection, di-

Table 4. Within-group changes in cancer markers in overweight and normal-weight patients after a 6-week lorazepam treatment

\begin{tabular}{|c|c|c|c|c|c|c|c|c|}
\hline & \multirow{2}{*}{ Baseline } & \multicolumn{2}{|c|}{ Overweight $(\mathrm{N}=9)$} & \multirow{2}{*}{$\mathrm{p}^{*}$} & \multirow{2}{*}{ Baseline } & \multicolumn{2}{|c|}{ Normal-weight $(\mathrm{N}=10)$} & \multirow{2}{*}{$\mathrm{p}^{*}$} \\
\hline & & Week 6 & $\mathrm{t}$ & & & Week 6 & $\mathrm{t}$ & \\
\hline ANG-2 & $485 \pm 149.2$ & $569.1 \pm 150.4$ & 4.129 & 0.003 & $671.2 \pm 158.7$ & $619.1 \pm 170$ & 2.232 & 0.052 \\
\hline sCD40L & $86.6 \pm 18.9$ & $94.8 \pm 13.2$ & 1.363 & 0.210 & $81.9 \pm 12.3$ & $87.5 \pm 19$ & 1.213 & 0.256 \\
\hline EGF & $25.9 \pm 6.6$ & $28.2 \pm 4.7$ & 1.140 & 0.287 & $23.9 \pm 4.4$ & $27.1 \pm 10.3$ & 1.27 & 0.236 \\
\hline Endoglin & $761.8 \pm 451.1$ & $578.3 \pm 226.3$ & 1.134 & 0.290 & $545.4 \pm 133.1$ & $618.1 \pm 283.5$ & 1.157 & 0.277 \\
\hline sFASL & $120.4 \pm 24.7$ & $137 \pm 24.2$ & 3.823 & 0.005 & $125.1 \pm 16.8$ & $125.9 \pm 23.1$ & 0.187 & 0.856 \\
\hline HB-EGF & $97 \pm 19.8$ & $110.4 \pm 14.1$ & 3.445 & 0.009 & $97.8 \pm 11.7$ & $98.3 \pm 19.3$ & 0.135 & 0.896 \\
\hline IGFBP & $1227 \pm 354$ & $1345 \pm 406$ & 1.276 & 0.238 & $1247 \pm 274$ & $1410 \pm 468$ & 1.337 & 0.214 \\
\hline IL-6 & $23.6 \pm 4.5$ & $26 \pm 3.4$ & 2.235 & 0.056 & $23.1 \pm 3.7$ & $23.2 \pm 4.5$ & 0.098 & 0.924 \\
\hline IL-8 & $5.9 \pm 1.1$ & $6.9 \pm 1.2$ & 3.750 & 0.006 & $6 \pm 1$ & $6.1 \pm 1.2$ & 0.678 & 0.515 \\
\hline IL-18 & $66.4 \pm 30.9$ & $63.3 \pm 37.9$ & 0.315 & 0.761 & $49 \pm 16.6$ & $52.4 \pm 15.8$ & 0.621 & 0.55 \\
\hline PAI & $4749 \pm 2437$ & $3692 \pm 1389$ & 1.071 & 0.315 & $3413 \pm 689$ & $3742 \pm 899$ & 0.908 & 0.388 \\
\hline PLGF & $26.4 \pm 7.4$ & $31.5 \pm 5.6$ & 3.471 & 0.008 & $26 \pm 7$ & $25.3 \pm 7.9$ & 0.634 & 0.542 \\
\hline TGF- $\alpha$ & $29.8 \pm 7.1$ & $35 \pm 5.4$ & 3.804 & 0.005 & $30.2 \pm 5$ & $30.4 \pm 7$ & 0.09 & 0.93 \\
\hline TNF- $\alpha$ & $15.3 \pm 3.6$ & $17.6 \pm 2.1$ & 2.544 & 0.035 & $15.8 \pm 3.2$ & $15.6 \pm 3.5$ & 0.223 & 0.828 \\
\hline $\mathrm{uPA}$ & $425.7 \pm 178.3$ & $535.6 \pm 202.2$ & 2.678 & 0.028 & $493.7 \pm 227$ & $582.4 \pm 307.5$ & 2.61 & 0.028 \\
\hline VEGF-A & $144.9 \pm 32.4$ & $160.7 \pm 23.3$ & 2.320 & 0.049 & $142.4 \pm 26.5$ & $147.5 \pm 28$ & 1.011 & 0.338 \\
\hline VEGF-C & $446.5 \pm 91.9$ & $509.1 \pm 42.8$ & 2.806 & 0.023 & $450.9 \pm 83.4$ & $447.8 \pm 84.6$ & 0.198 & 0.848 \\
\hline VEGF-D & $278.3 \pm 74.4$ & $309.7 \pm 77.2$ & 3.275 & 0.011 & $269.9 \pm 29.3$ & $268.1 \pm 34.9$ & 0.261 & 0.8 \\
\hline
\end{tabular}

*differences in cancer markers after six weeks of lorazepam treatment were analyzed with paired-samples t-tests. ANG-2: angiopoietin-2, sCD40L: soluble CD40 ligand, EGF: epidermal growth factor, sFASL: soluble Fas ligand, HB-EGF: heparin-binding EGF-like growth factor, IGFBP: insulin-like growth factor-binding protein, IL: interleukin, PAI: plasminogen activator inhibitor, PLGF: placental growth factor, TGF: transforming growth factor, TNF: tumor necrosis factor, uPA: urokinase-type plasminogen activator, VEGF: vascular endothelial growth factor

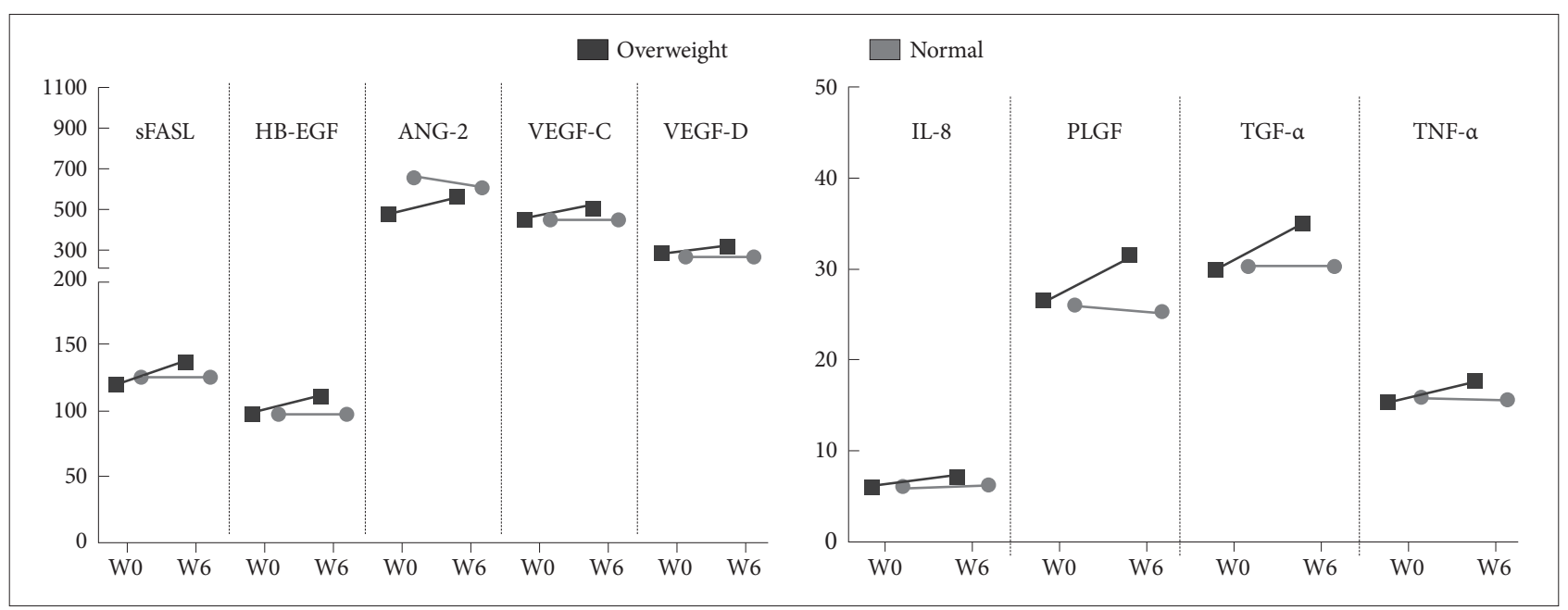

Figure 1. Distinct pattern of caner biomarker changes between the overweight and the normal-weight patients. After six weeks of treatment with lorazepam, the nine cancer biomarkers were significantly increased in the overweight patients but not in the normal-weight patients. sFASL: soluble Fas ligand, HB-EGF: heparin-binding EGF-like growth factor, ANG-2: angiopoietin-2, VEGF: vascular endothelial growth factor, IL: interleukin, PLGF: placental growth factor, TGF: transforming growth factor, TNF: tumor necrosis factor. 
agnosis, and treatment. Finally, this study lacks a randomized control group, so the effects of time-in-hospital cannot be excluded. Therefore, further studies with prospective randomization to a control group are encouraged.

Evidence from basic research cannot be directly generalized to humans. To date, the link between benzodiazepines and carcinogenesis is suggestive but not conclusive. In our study, lorazepam is assumed work in concert with adiposity, triggering the carcinogenesis as evidenced by changes in cancer biomarkers. Epidemiological studies addressing this issue should consider the potential modulator contributing to benzodiazepine-associated carcinogenesis. Further research with a prospective follow-up design to explore the long-term effects of benzodiazepines on carcinogenesis is strongly encouraged.

\section{Acknowledgments}

This study was provided by the Civilian Administration Division of Beitou Branch, Tri-Service General Hospital, National Defense Medical Center (TSGH-BT-105-001).

\section{REFERENCES}

1. Rudolph U, Knoflach F. Beyond classical benzodiazepines: novel therapeutic potential of GABAA receptor subtypes. Nat Rev Drug Discov 2011;10:685-697.

2. Kripke DF. Hypnotic drug risks of mortality, infection, depression, and cancer: but lack of benefit. F1000Res 2016;5:918.

3. Miyawaki I, Moriyasu M, Funabashi H, Yasuba M, Matsuoka N. Mechanism of clobazam-induced thyroidal oncogenesis in male rats. Toxicol Lett 2003;145:291-301.

4. Iida M, Anna CH, Hartis J, Bruno M, Wetmore B, Dubin JR, et al. Changes in global gene and protein expression during early mouse liver carcinogenesis induced by non-genotoxic model carcinogens oxazepam and Wyeth-14,643. Carcinogenesis 2003;24:757-770.
5. Kripke DF, Langer RD, Kline LE. Hypnotics' association with mortality or cancer: a matched cohort study. BMJ Open 2012;2:e000850.

6. Iqbal U, Nguyen PA, Syed-Abdul S, Yang HC, Huang CW, Jian WS, et al. Is long-term use of benzodiazepine a risk for cancer? Medicine (Baltimore) 2015;94:e483.

7. Kunert-Radek J, Stepien H, Pawlikowski M. Inhibition of rat pituitary tumor cell proliferation by benzodiazepines in vitro. Neuroendocrinology 1994;59:92-96.

8. Kim DH, Lee JT, Lee IK, Ha JH. Comparative anticancer effects of flavonoids and diazepam in cultured cancer cells. Biol Pharm Bull 2008;31:255-259.

9. Pottegard A, Friis S, Andersen M, Hallas J. Use of benzodiazepines or benzodiazepine related drugs and the risk of cancer: a populationbased case-control study. Br J Clin Pharmacol 2013;75:1356-1364.

10. Hanahan D, Weinberg RA. The hallmarks of cancer. Cell 2000;100:57-70.

11. Renehan AG, Tyson M, Egger M, Heller RF, Zwahlen M. Body-mass index and incidence of cancer: a systematic review and meta-analysis of prospective observational studies. Lancet 2008;371:569-578.

12. Calle EE, Rodriguez C, Walker-Thurmond K, Thun MJ. Overweight, obesity, and mortality from cancer in a prospectively studied cohort of U.S. adults. N Engl J Med 2003;348:1625-1638.

13. Guh DP, Zhang W, Bansback N, Amarsi Z, Birmingham CL, Anis AH. The incidence of co-morbidities related to obesity and overweight: a systematic review and meta-analysis. BMC Public Health 2009;9:88.

14. Richens JL, Urbanowicz RA, Metcalf R, Corne J, O’Shea P, Fairclough L. Quantitative validation and comparison of multiplex cytokine kits. J Biomol Screen 2010;15:562-568.

15. Khandekar MJ, Cohen P, Spiegelman BM. Molecular mechanisms of cancer development in obesity. Nat Rev Cancer 2011;11:886-895.

16. Renehan AG, Zwahlen M, Egger M. Adiposity and cancer risk: new mechanistic insights from epidemiology. Nat Rev Cancer 2015;15:484-498.

17. Brambilla G, Carrozzino R, Martelli A. Genotoxicity and carcinogenicity studies of benzodiazepines. Pharmacol Res 2007;56:443-458.

18. Iakovidou-Kritsi Z, Akritopoulou K, Ekonomopoulou MT, Mourelatos D. In vitro genotoxicity of two widely used benzodiazepines: alprazolam and lorazepam. Arist Uni Med J 2009;36:39-44.

19. Kripke DF. Possibility that certain hypnotics might cause cancer in skin. J Sleep Res 2008;17:245-250. 